\title{
Administration of nimotuzumab combined with cisplatin plus 5-fluorouracil as induction therapy improves treatment response and tolerance in patients with locally advanced nasopharyngeal carcinoma receiving concurrent radiochemotherapy: a multicenter randomized controlled study
}

Ying Lu', Dagui Chen ${ }^{1}$, Jinhui Liang ${ }^{2}$, Jianquan Gao², Zhanxiong Luo ${ }^{3}$, Rensheng Wang ${ }^{4}$, Wenqi Liư ${ }^{5}$, Changjie Huang ${ }^{6}, X_{\text {Xejian Ning }}^{7}$, Meilian Liu ${ }^{8}$ and Haixin Huang ${ }^{1 *}$

\section{Abstract}

Background: Nimotuzumab (NTZ) is an anti-EGFR monoclonal antibody. However,the effect of targeted drugs combined with induction therapy in locally advanced nasopharyngeal carcinoma remains unclear. The aim of this study is to investigate the safety and efficacy of NTZ combined with cisplatin plus 5-fluorouracil (PF) as induction regimen in locally advanced nasopharyngeal carcinoma (NPC) patients receiving concurrent radiochemotherapy.

Methods: This was a multicenter randomized controlled study performed in eight Guangxi hospitals in 2015-2017. Eligible patients with NPC were randomized into nimotuzumab/PF (NPF group) and docetaxel/PF (DPF group) regimens, respectively, as induction therapy. After 2 cycles of induction therapy, all patients received cisplatin and concurrent intensity modulated radiation therapy (IMRT). Then, the two groups were compared for safety and efficacy.

\footnotetext{
*Correspondence: 13507726193@163.com

'Department of Oncology, the Fourth Affiliated Hospital of Guangxi Medical

University, Liuzhou, China

Full list of author information is available at the end of the article
}

(c) The Author(s). 2019 Open Access This article is distributed under the terms of the Creative Commons Attribution 4.0 International License (http://creativecommons.org/licenses/by/4.0/), which permits unrestricted use, distribution, and reproduction in any medium, provided you give appropriate credit to the original author(s) and the source, provide a link to the Creative Commons license, and indicate if changes were made. The Creative Commons Public Domain Dedication waiver (http://creativecommons.org/publicdomain/zero/1.0/) applies to the data made available in this article, unless otherwise stated. 
(Continued from previous page)

Results: A total of 118 patients with stage III-IVa NPC were assessed, with 58 and 60 in the NPF and DPF groups, respectively. Compared with DPF treatment, NPF induction therapy showed a more pronounced effect on cervical lymph nodes $(P=0.036)$, with higher response rate $(R R)(81 \%$ vs 60\%). Compared with the DPF group, the NPF group showed significantly reduced leukopenia, neutropenia and gastrointestinal reactions (all $P<0.05$ ); rash only appeared in the NPF group, but all cases were grade 1. During concurrent treatment with radiotherapy and chemotherapy, the NPF group showed better tolerance to radiotherapy and chemotherapy; neutropenia, anemia, gastrointestinal reactions, oral mucositis and radiation dermatitis in the NPF group were significantly reduced $(P<$ 0.05). The expression rate of EGFR was $94.9 \%$ (112/118). Compared with the DPF group, patients with EGFR expression in the NPF group showed better response (77.8\% vs 63.0\%, $P=0.033$ ).

Conclusion: For locally advanced NPC patients receiving follow-up cisplatin and IMRT, nimotuzumab/PF for induction therapy has better lymph node response rate and milder adverse reactions than the DPF regimen. In addition, the patients have better tolerance in subsequent concurrent radiotherapy and chemotherapy; however, long-term efficacy needs further follow-up evaluation.

Trial registration: The registration number of the clinical trial is ChiCTR-OIC-16008201 and retrospectively registered on March 31, 2016.

Keywords: Locally advanced nasopharyngeal carcinoma, EGFR monoclonal antibody, Induction therapy, Curative effect, Adverse reaction

\section{Background}

Nasopharyngeal carcinoma (NPC) is a head and neck cancer with a unique biological behavior; unlike other head and neck tumors, NPC has higher sensitivity to radiotherapy and chemotherapy [1-3]. Therefore, radiochemotherapy is the main treatment option for locally advanced NPC. With the application of intensity modulated radiation therapy (IMRT), distant metastasis has become a major factor affecting prognosis in NPC [3]. Induction therapy may reduce the micrometastasis of NPC, better radiotherapeutic conditions for locally advanced nasopharyngeal carcinoma (especially in patients with giant lesions), and improve patient survival and prognosis [4]. Based on concurrent radiotherapy and chemotherapy (CCRT), induction chemotherapy (IC) could increase the 5-year absolute benefit rates of progression-free survival (PFS) and distance control (DC) by 4.2 and $8.7 \%$, respectively reduce the risk of cancer-related death by $4.8 \%$ [5], and improve the overall survival (OS) rate of patients [6]. IC-CCRT is the most effective $\mathrm{DC}$ regimen $(\mathrm{HR}=0.44,95 \% \mathrm{CI} \quad 0.27-0.71$; Pscore 95\%) in the comprehensive treatment of NPC. It improves OS (HR $=0.81,95 \%$ CI 0.63-1.04; P-score 63\%) and PFS $(\mathrm{HR}=0.68,95 \% \mathrm{CI}$ 0.54-0.85; P-score 95\%) [5]. Therefore, induction chemotherapy combined with concurrent radiochemotherapy is considered an effective therapeutic mode for locally advanced NPC [4, 7], but the optimal induction therapy remains uncertain.

The DPF regimen consisting of docetaxel, cisplatin and 5-fluorouracil shows a higher objective remission rate (ORR) compared with the cisplatin plus 5fluorouracil (PF) regimen. It is recommended by category I of NCCN guidelines for diagnosis and treatment of head and neck squamous cell carcinoma [8, 9], Induction therapy with the DPF regimen is also used clinically for NPC patients, in whom the ORRs of primary lesions and cervical lymph nodes after DPF induction chemotherapy could reach 92.9\% [10]. Based on concurrent radiochemotherapy, DPF chemotherapy effectively improves 3-year OS (86\% vs 92\%, $P=0.029)$ and distant metastasis-free survival (DMFS) $(83 \%$ vs $90 \%, P=0.031)$ [11]. In N2-3 NPC patients, the 3-year distant metastasis rate decreases by $26 \%(P=0.08)$ while OS increases by $25 \%(P=0.21)$ [12]. Compared with induction chemotherapy such as administration of docetaxel combined with cisplatin (TP) or PF, the DPF regimen alone significantly increases PFS (HR $=0.70 ; 95 \%$ CI 0.49-0.95), OS (HR $=0.59 ; 95 \% C I$ 0.37-0.92) [6]. However, while the DPF regimen achieves higher efficacy, treatment-related adverse reactions also increase significantly, especially the incidence of grade 3-4 neutropenia whose rate could reach $42 \%[11,13]$, which hampers the clinical application of DPF.

With the gradual development of molecular biology research, molecular targeted therapy has become a research hotspot in cancer therapy. The expression rate of epidermal growth factor receptor (EGFR) in nasopharyngeal carcinoma is $68-89 \%$, which is much higher than that of other solid tumors [14]. Meanwhile, EGFR expression is closely related to prognosis in NPC [15-17]. Overexpression of EGRF significantly increases the risk of adverse prognosis of NPC OS $(\mathrm{HR}=1.86,95 \% \mathrm{CI}$ $1.25-2.77 ; P=0.000)$, disease-free survival (DFS) $(\mathrm{HR}=$ 2.25 , 95\%CI 1.66-3.04; $P=0.000)$, locoregional recurrence free survival (LRFS) $(\mathrm{HR}=2.93,95 \% \mathrm{CI} 1.71-5.02$; $P=0.000)$ [15]. In patients with locally advanced NPC 
receiving IMRT, combined anti-EGFR receptor therapy may be a more effective treatment strategy [18].

Nimotuzumab (NTZ) is a humanized monoclonal antibody [19, 20]. Compared with cetuximab (CTX), NTZ is human-derived and highly selective, with a long half-life. It competitively inhibits the binding of endogenous ligands to EGFR and blocks the downstream signal transduction pathway mediated by EGFR, thereby inhibiting the proliferation of tumor cells, promoting apoptosis of tumor cells, suppressing angiogenesis and increasing radiosensitivity to chemotherapy; meanwhile, it has few adverse reactions and low incidence of rash. Therefore, whether combined with CCRT or applied as an induction therapeutic, skin rash and mucosal reactions of nimotuzumab are significantly improved compared with those of cetuximab. Retrospective analysis suggested that sequential concurrent radiochemotherapy after induction chemotherapy combined with NTZ is effective and well tolerated in the treatment of locally advanced NPC [21]. In addition, it was demonstrated that NTZ combined with concurrent chemoradiotherapy is beneficial in the treatment of locally advanced NPC, with limited toxicity and good-tolerability [22]. However, there is a lack of prospective analysis of nimotuzumab for induction therapy in locally advanced NPC. Therefore, the current randomized controlled study aimed to assess the safety and efficacy of NTZ combined with PF as induction regimen in locally advanced NPC patients receiving concurrent radiochemotherapy.

\section{Methods}

\section{Patients}

This randomized controlled study assessed NPC patients receiving initial treatment in eight hospitals in Guangxi (Oncology Department of the Fourth Affiliated Hospital of Guangxi Medical University, Radiotherapy Department of Wuzhou Red Cross Hospital, Radiotherapy Department of Liuzhou People's Hospital, Radiotherapy Department of the First Affiliated Hospital of Guangxi Medical University, Radiotherapy Department of the Second Affiliated Hospital of Guangxi Medical University, Oncology Department of Nanning Second People's Hospital, Oncology Department of Liuzhou Traditional Chinese Medicine Hospital, Radiotherapy Department of the affiliated hospital of Guilin Medical College) from January 2015 to December 2017.

Inclusion criteria were: 1) 18-70 years old; 2) undifferentiated non-keratinizing nasopharyngeal carcinoma pathologically diagnosed by biopsy; 3 ) clinical stage IIIIVa (08 Chinese stage); 4) KPS score $\geq 70$; 5) serum hemoglobin $\geq 10 \mathrm{mg} / \mathrm{dL}$, platelet $\geq 10,000 / \mathrm{mu} \mathrm{L}$, and absolute neutrophil count $\geq 1500 / \mathrm{mu} \mathrm{L}$; 6) serum creatinine $\leq 1.5$ times UNL or creatinine clearance rate $\geq 60 \mathrm{ml} /$ min; bilirubin $\leq 1.5$ times UNL and AST (SGOT) and
ALT $(\mathrm{SGPT}) \leq 1.5$ times UNL; 8) estimated survival time $\geq 6$ months.

Exclusion criteria were: 1) previous diagnosis of malignant tumors; 2) previous radiotherapy, chemotherapy, or targeted therapy; 3 ) radiotherapy or chemotherapy contraindications; 4) allergy to any study drug.

The study was approved by the Ethics Committee of the Fourth Affiliated Hospital of Guangxi Medical University (PJK2015201). The registration number of the clinical trial is ChiCTR-OIC-16008201, and all patients provided signed informed consent obtained was written.

\section{Treatments}

In this open-label trial, allocation concealment was performed before eligible patients were randomly divided into two groups: NPF (induction therapy with nimotuzumab combined with PF; nimotuzumab $200 \mathrm{mg} /$ time/ week by intravenous drip, cisplatin $75 \mathrm{~g} / \mathrm{m}^{2}$ by intravenous drip at $\mathrm{d} 1$, and continuous infusion of 5 -fluorouracil $750 \mathrm{mg} / \mathrm{m}^{2} /$ day at $\mathrm{d} 1-5$; repeated every 3 weeks) and DPF (induction chemotherapy; docetaxel $75 \mathrm{~g} / \mathrm{m}^{2}$ by intravenous drip at $\mathrm{d} 1$, cisplatin $75 \mathrm{~g} / \mathrm{m}^{2}$ by intravenous drip at $\mathrm{d} 1$, and continuous infusion of 5 -fluorouracil $750 \mathrm{mg} / \mathrm{m}^{2} / \mathrm{d}$ at $\mathrm{d} 1-5$; repeated every 3 weeks) groups. After 2 cycles of induction therapy, cisplatin $\left(80 \mathrm{~g} / \mathrm{m}^{2}\right.$ by intravenous drip at $\mathrm{d} 1$, repeated every 3 weeks, for a total of 3 cycles) was administered to both groups concurrently with intensity modulated radiation therapy (IMRT). G-CSF was not administered prophylactically before the first induction therapy or concurrent chemotherapy; in patients with grade 4 neutropenia, prophylactic G-CSF leucocyte-raising therapy was used during the follow-up cycle. In patients who still showed intolerability, chemotherapeutic drug dosage was reduced by $15 \%$ according to the principle of reduction of chemotherapeutic drugs. All chemotherapy cycles routinely included antiemetic treatment, and the follow-up cycle antiemetic regimen was adjusted according to gastrointestinal reactions.

All patients were treated by IMRT. The range of tumors was determined by magnetic resonance (MR), and the target area was delineated and planned by enhanced CT. According to the actual situation of each center, the planning target volume (PTV) formed by expanding 3-5 $\mathrm{mm}$ from each target area was administered a prescription dose: nasopharyngeal tumor volume (GTVnx) and cervical lymph node volume (GTVnd) at 69.69-70.06 Gy and 64.17-70.06 Gy, respectively; high-risk area of primary focus (CTV1) at 66.03 Gy; low-risk area of primary focus and cervical lymph node drainage area (CTV2) at 50.4-54.25 Gy. The number of segmentations was 31-33 times, 5 times a week. According to the requirements of RTOG 0615 and RTOG 0225, organ-threatening dose and planning were assessed. 


\section{Evaluation indexes}

The primary endpoints were the efficacy of induction therapy and adverse reactions to induction therapy. The secondary endpoints were immediate and 3-month follow-up effects of the whole therapy and adverse reactions to concurrent chemoradiotherapy. The acute and late side effects of radiotherapy were evaluated according to RTOG acute and late radiation reaction scoring criteria, and the adverse reactions related to chemotherapy were assessed according to NCI-CTC AE3.0 classification criteria of common adverse drug reactions. The curative effects were based on Response Evaluation Criteria in Solid Tumors (RECIST 1.1) [23] and included complete response (CR; no detectable cancer after your treatment), partial response (PR; at least 30\% decrease in total lesion area), stable disease (SD; no change or decrease of total lesion area below 30\%) and progressive disease (PD; increase in total lesion area).

\section{EGFR detection}

EGFR expression in nasopharyngeal carcinoma tissues was evaluated by immunohistochemical staining. The tumor tissues were fixed with $10 \%$ formaldehyde, paraffin embedded and sliced into 4- $\mu \mathrm{m}$ sections. After conventional dewaxing, endogenous peroxidase activity was blocked by treatment with hydrogen peroxide, followed by antigen retrieval via heating. Then, the samples were successively incubated with primary (overnight at $4{ }^{\circ} \mathrm{C}$ ) and biotinylated secondary $\left(37^{\circ} \mathrm{C}\right.$ for $\left.30 \mathrm{~min}\right)$ antibodies, and treated with $\mathrm{DAB}$ for $10 \mathrm{~min}$. After dehydration with graded ethanol, counterstaining was performed with hematoxylin. The range and intensity of staining were observed and recorded under a microscope. Phosphate buffer saline was used as a negative control, and known positive samples were used as positive controls. Grading was performed according to staining intensity and the percentage of positive cells. Positive signals were dark blue-purple granules in the cell membrane or cytoplasm of the tumor cells; positive cells were grouped into 0$4 \%, 5-24 \%, 25-49 \%, 50-75$ and $>75 \%$. Two deputy directors of pathology interpreted the results independently.

\section{Statistical methods}

In the current non-inferiority study, the efficacy of induction therapy was the primary point for noninferiority and RR decreases less than $5 \%$ as noninferiority margin. We used an alpha of 0.05 and a beta of 0.20 . Considering a dropout rate of $10 \%$, we found that 60 was an appropriate sample size for each group. Therefore, a total of 120 patients were enrolled. IBM SPSS 19.0 was used for statistical analysis. Measurement data were analyzed using $t$ test, while count data were compared by the $\mathrm{X}^{2}$ test. Differences were considered significant at $p<0.05$.

\section{Results \\ Baseline patient characteristics}

From May 2015 to November 2017, a total of 118 patients were enrolled in the current study. The study flowchart was shown in Fig. 1. The average age of all patients was 44 years (22-68 years). There were 98 males and 20 females, indicating a male to female ratio of 4.9. A total of 66 cases had stage III disease while 52 had stage IVa. The NPF and DPF groups comprised 58 and 60 cases, respectively. There were no significant differences in baseline characteristics, including age, sex, and clinical stage between the two groups $(P>0.05)$ (Table 1).

\section{Therapeutic effects}

By September 2018, all patients had completed induction therapy and therapeutic effects were evaluated. In the induction stage, compared with the DPF group, NPF induction therapy had more pronounced effects on cervical lymph nodes $(P=0.036)$ and $\mathrm{RR}(\mathrm{CR}+\mathrm{PR})(81 \%$ vs $60 \%)$. There were no significant differences in nasopharyngeal lesions and overall efficacy $(P=0.446, P=$ 0.143 ) between the two groups. One case of lung metastasis after induction chemotherapy in the DPF group exited the study. A total of 117 patients were further treated with cisplatin and concurrent IMRT, including 58 and 59 in the NPF and DPF groups, respectively. There were no significant differences between the two groups $(P=0.449$ and $P=0.409)$ in immediate efficacy evaluation and 3-month efficacy evaluation at the end of the whole course of treatment (Table 2).

\section{Adverse reactions}

In the induction stage, the main adverse reactions in the two groups were grade 1-2 leukopenia, neutropenia and gastrointestinal reactions. Compared with the DPF group, the NPF group showed significantly reduced leucopenia, neutropenia and gastrointestinal reactions ( $P=0.037, P=0.018$ and $P=0.032$, respectively). Rashes only appeared in the NPF group, and all were grade 1 ; after NTZ treatment, rashes could disappear spontaneously. There were no significant differences in hemoglobin decrease, thrombocytopenia, liver or kidney impairment, and oral mucositis $(P>0.05)$. In the concurrent radiotherapy and chemotherapy phase, the NTP group showed better treatment tolerance. Neutropenia, anemia, gastrointestinal reactions, oral mucositis and radiation dermatitis were significantly reduced in the NTP group compared with DPF group $(P=0.033, P=0.049$, $P=0.037, \quad P=0.020$ and $P=0.035$, respectively). Leukopenia, thrombocytopenia, and liver and kidney 


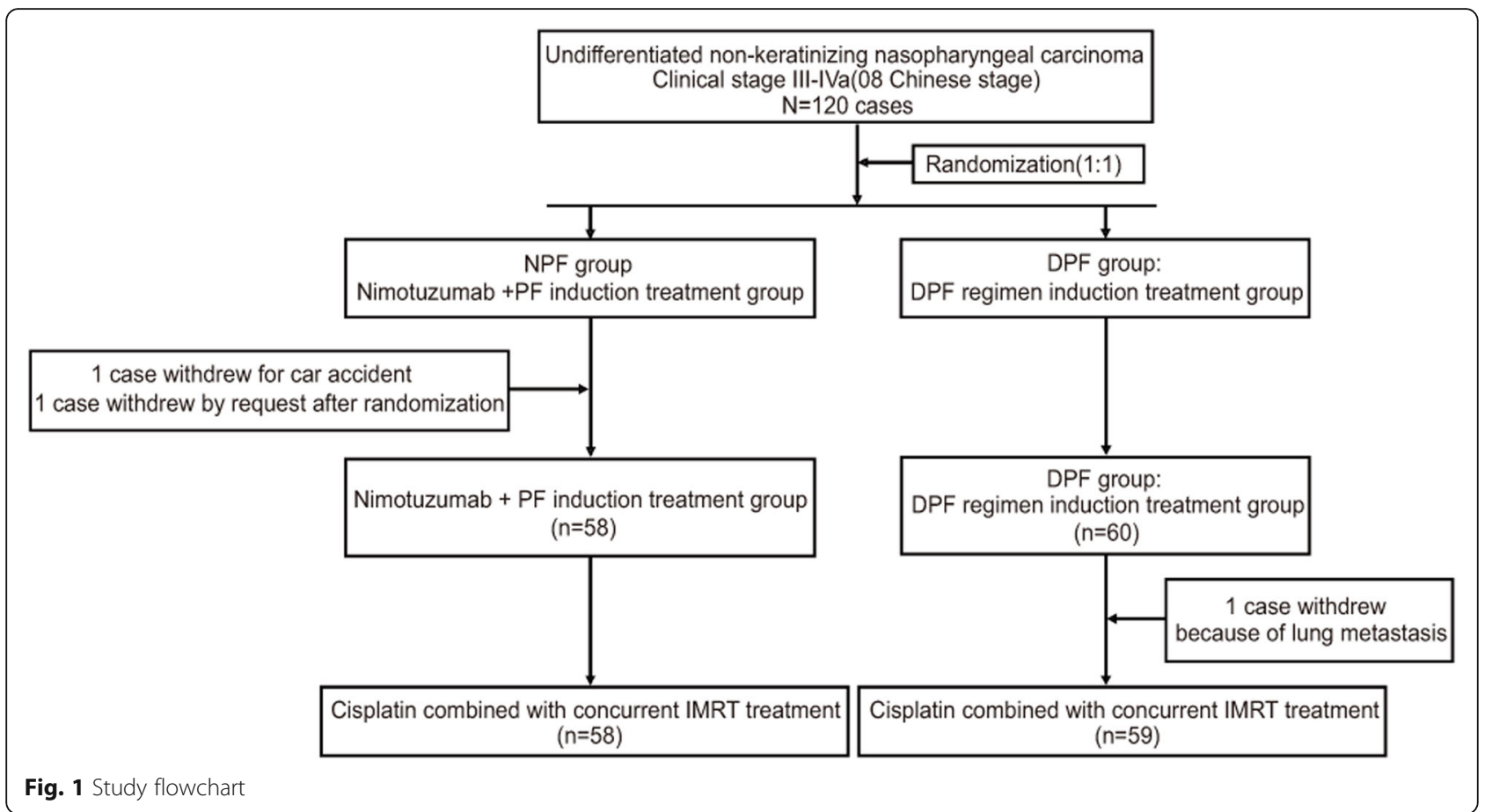

Table 1 Clinical characteristics of the two groups

\begin{tabular}{|c|c|c|c|c|}
\hline \multirow[t]{2}{*}{ Characteristics } & \multicolumn{3}{|c|}{ No. of patients (\%) } & \multirow[t]{2}{*}{$P$} \\
\hline & $\begin{array}{l}\text { Total } \\
(n=118)\end{array}$ & $\begin{array}{l}\text { NPF group } \\
(n=58)\end{array}$ & $\begin{array}{l}\text { DPF group } \\
(n=60)\end{array}$ & \\
\hline Median age (range) & $44(22-68)$ & $43(22-65)$ & $45(22-68)$ & 0.214 \\
\hline \multicolumn{5}{|l|}{ Gender } \\
\hline Males & $98(83.1)$ & $47(81.0)$ & $51(85.0)$ & \multirow[t]{2}{*}{0.371} \\
\hline Females & 20(16.9) & $11(19.0)$ & $9(15.0)$ & \\
\hline \multicolumn{5}{|c|}{ Clinical stage (China, 2008) } \\
\hline III & $66(55.9)$ & $34(58.6)$ & $32(53.3)$ & \multirow[t]{2}{*}{0.347} \\
\hline $\mathrm{IVa}$ & $52(44.1)$ & $24(41.4)$ & $28(46.7)$ & \\
\hline \multicolumn{4}{|l|}{ T stage } & \multirow[t]{5}{*}{0.436} \\
\hline $\mathrm{T} 1$ & $6(5.1)$ & $3(5.1)$ & $3(5.0)$ & \\
\hline $\mathrm{T} 2$ & $20(16.9)$ & $12(20.7)$ & $8(13.3)$ & \\
\hline T3 & $57(48.3)$ & $27(46.6)$ & $30(50.0)$ & \\
\hline T4 & $35(29.7)$ & $16(27.6)$ & 19(31.7) & \\
\hline \multicolumn{4}{|l|}{ N stage } & \multirow[t]{5}{*}{0.585} \\
\hline No & $3(25.4)$ & $2(3.4)$ & $1(1.6)$ & \\
\hline $\mathrm{N} 1$ & $27(22.9)$ & $15(25.9)$ & $12(20.0)$ & \\
\hline N2 & $67(56.9)$ & $30(51.7)$ & $37(61.7)$ & \\
\hline N3 & $21(17.8)$ & $11(19.0)$ & $10(16.7)$ & \\
\hline
\end{tabular}

functions were also improved, but the differences were not statistically significant $(P>0.05)$ (Table 3$)$.

\section{Association of EGFR expression with the efficacy of induction therapy}

The overall expression rate of EGFR was 94.9\% (112/ $118)$, including $94.8 \%(55 / 58)$ and $95.5 \%(57 / 60)$ in the NPF and DPF groups, respectively. There was no significant difference in EGFR expression levels between the two groups $(P=0.058)$ (Table 4$)$. EGFR expression was not significantly correlated with the efficacy of induction chemotherapy with DPF $(P=0.090)$, but significantly affected the efficacy of induction therapy combined with nimotuzumab $(P=0.015)$; compared with chemotherapy, induction therapy combined with nimotuzumab had better response $(77.8 \%$ vs $63.0 \%, P=0.033)$., as shown in Table 5.

\section{Discussion}

This study assessed the effectiveness of NTZ combined with PF as induction regimen in locally advanced NPC cases receiving concurrent radiochemotherapy, and demonstrated that nimotuzumab combined with PF for induction therapy has better lymph node response rate and milder adverse reactions compared with the DPF regimen. In addition, the patients showed improved tolerance in subsequent concurrent radiotherapy and chemotherapy.

In a study by Chua DT [24] EGFR was shown to be expressed in $89 \%$ of nasopharyngeal carcinoma cases, and high EGFR expression is considered an independent 
Table 2 Therapeutic effects in the two groups

\begin{tabular}{|c|c|c|c|c|c|c|c|c|c|c|}
\hline \multirow[t]{3}{*}{$\begin{array}{l}\text { Therapeutic } \\
\text { effect }^{\mathrm{a}}\end{array}$} & \multicolumn{6}{|c|}{$\begin{array}{l}\text { After induction therapy } \\
(n=118)\end{array}$} & \multirow{2}{*}{\multicolumn{2}{|c|}{$\begin{array}{l}\text { After concurrent } \\
\text { radiochemotherapy } \\
(n=117)^{b}\end{array}$}} & \multirow{2}{*}{\multicolumn{2}{|c|}{$\begin{array}{l}3 \text { months after the } \\
\text { treatment } \\
(n=117)\end{array}$}} \\
\hline & \multicolumn{2}{|c|}{$\begin{array}{l}\text { Nasopharyngeal lesions } \\
(\%)\end{array}$} & \multicolumn{2}{|c|}{ Lymph node lesions (\%) } & \multicolumn{2}{|c|}{$\begin{array}{l}\text { Total(\%)(nasopharyngeal } \\
\text { and lymph node lesions) }\end{array}$} & & & & \\
\hline & $\begin{array}{l}\text { NPF group } \\
(n=58)\end{array}$ & $\begin{array}{l}\text { DPF group } \\
(n=60)\end{array}$ & $\begin{array}{l}\text { NPF group } \\
(n=58)\end{array}$ & $\begin{array}{l}\text { DPF group } \\
(n=60)\end{array}$ & $\begin{array}{l}\text { NPF group } \\
(n=58)\end{array}$ & $\begin{array}{l}\text { DPF group } \\
(n=60)\end{array}$ & $\begin{array}{l}\text { NPF group } \\
(n=58)\end{array}$ & $\begin{array}{l}\text { DPF group } \\
(n=59)\end{array}$ & $\begin{array}{l}\text { NPF group } \\
(n=58)\end{array}$ & $\begin{array}{l}\text { DPF group } \\
(n=59)\end{array}$ \\
\hline$C R$ & $2(3.4)$ & $0(0)$ & $5(8.6)$ & $1(1.7)$ & $2(3.4)$ & $0(0)$ & $39(67.2)$ & $38(64.4)$ & $51(87.9)$ & $50(84.7)$ \\
\hline$P R$ & 33(56.9) & $31(51.7)$ & $42(72.4)$ & $35(58.3)$ & $39(67.2)$ & $37(61.7)$ & 19(32.8) & 21(35.6) & $7(12.1)$ & $9(15.3)$ \\
\hline SD & 23(39.7) & $29(48.3)$ & 11(19.0) & $24(40.0)$ & 17(29.3) & $22(36.7)$ & $0(0)$ & $0(0)$ & $0(0)$ & $0(0)$ \\
\hline PD & $0(0)$ & $0(0)$ & $0(0)$ & $0(0)$ & $0(0)$ & $1(1.7)$ & $0(0)$ & $0(0)$ & $0(0)$ & $0(0)$ \\
\hline P & 0.446 & & 0.036 & & 0.143 & & 0.499 & & 0.409 & \\
\hline
\end{tabular}

${ }^{a} C R$ Complete remission, $P R$ Partial remission, $S D$ Stable disease, $P D$ Progressive disease

${ }^{\mathrm{b}}$ After induction therapy, 1 patient in the DPF group showed distant metastasis and withdrew from the study

prognostic factor for local control, non-recurrence and disease-related survival in stage III-IV NPC. In the latter report, $72 \%$ of patients with EGFR expression (>25\%) showed significant adverse prognosis after induction chemotherapy and radiotherapy. It was therefore suggested that anti-EGFR therapy might be necessary to improve prognosis in locally advanced NPC with high EGFR expression to increase clinical benefits. In the present study, $94.9 \%$ of NPC patients expressed EGFR, including $77.7 \%$ whose EGFR expression exceeded 25\%, corroborating Chua's study [24] The efficacy of induction therapy combined with anti-EGFR was related to EGFR $(P=0.015)$. Meanwhile, supplementing anti-EGFR monoclonal antibody significantly affected the efficacy of induction therapy $(P=0.033)$, suggesting that induction therapy combined with anti-EGFR therapy is feasible.

One of the aims of induction therapy is to effectively alleviate the lesions and create improved radiotherapy conditions for NPC, especially in patients with giant lesions, achieving better prognosis. For instance, in NPC patients undergoing follow-up CCRT, 5-year OS rates in the CR, PR and SD subgroups after induction chemotherapy were shown to be $100,79.4$ and $60 \%$, respectively. The efficacy of induction therapy may therefore affect patient survival and prognosis [25]. It was reported that induction therapy with taxanes significantly increases ORR $(\mathrm{OR}=4.57,95 \% \mathrm{CI} 1.14-18.30, P=0.032$, $\mathrm{z}=2.15)$ [26] compared with the non-taxane regimen. However, in this study, induction therapy with NPF had higher RR in lymph node lesions $(81.0 \%$ vs $60 \%$ ) compared with DPF. These findings suggest that induction therapy combined with EGFR is more effective in alleviating lesions, creating better radiotherapy conditions and improving survival and prognosis in NPC with high EGFR expression.

With the application of IMRT, distant metastasis has become a major factor affecting prognosis in NPC. Meanwhile, induction therapy may reduce the micrometastasis of locally advanced NPC, and is considered an effective control scheme for distant metastasis in various comprehensive treatment regimens, improving PFS and OS [5]. Based on concurrent radiochemotherapy, the DPF regimen was added to induce chemotherapy, which effectively increased the 3-year OS $(P=0.029)$ and DMFS $(P=0.031)$ [11]. Meanwhile, the 3-year distant metastasis rate of NPC patients with N2-3 disease decreased by $26 \%(P=0.08)$, while OS increased by $25 \%$ $(P=0.21)$ [12]. Among the various induction therapies commonly used in clinic, the PF regimen significantly reduces the risk of adverse prognosis of PFS $(\mathrm{HR}=0.75$; 95\%CI, 0.56-0.99), whereas the DPF regimen increases the risk of adverse prognosis of OS while improving PFS $(\mathrm{HR}=0.59 ; 95 \% \mathrm{CI}, 0.37-0.92)$ [6]. However, adding EGFR monoclonal antibody based on DPF does not significantly increase the survival advantage of NPC patients. NTZ combined with DPF does not improve 5year OS (89.9\% vs $93.3 \%$ ) and PFS (79.3\% vs $82.1 \%)$ compared with NTZ combined with PF [21]. Therefore, EGFR combined with PF may represent a more economical and effective induction therapeutic regimen for NPC. As shown above, there was no significant difference between the NPF and DPF groups during immediate and 3-month curative effect evaluation. Although long-term survival data for NPF induction therapy and DPF induction chemotherapy were not available in this study, $\mathrm{N}$ stage is the main prognostic factor of DMFS and OS in nasopharyngeal carcinoma. A higher regional lymph node response rate $(P=0.036)$ after NPF induction therapy would help control regional lymph noderelated distant metastasis and improve survival. It is worth assessing long-term survival after treatment with the NPF regimen.

On the other hand, induction therapy by NTZ combined with PF reduces the intensity of chemotherapy compared with DPF, which is helpful in improving hematological toxicity that restricts the clinical application of DPF. Adverse reactions caused by induction chemotherapy, especially increased systemic toxicity, 
Table 3 Adverse reactions in the two groups

\begin{tabular}{|c|c|c|c|c|c|c|}
\hline \multirow[t]{2}{*}{ Adverse reaction } & \multicolumn{3}{|l|}{$\begin{array}{l}\text { Induction therapy stage } \\
(n=118)(\%)\end{array}$} & \multicolumn{3}{|l|}{$\begin{array}{l}\text { Concurrent radiochemotherapy stage } \\
(n=117)(\%)\end{array}$} \\
\hline & NPF group $(n=58)$ & DPF group $(n=60)$ & $P$ & NPF group $(n=58)$ & DPF group $(n=59)$ & $P$ \\
\hline \multicolumn{2}{|l|}{ Leukocytopenia } & & 0.037 & & & 0.090 \\
\hline 0 & $20(34.5)$ & $17(28.3)$ & & $14(24.1)$ & $11(18.6)$ & \\
\hline 1 & $28(48.3)$ & $21(35.0)$ & & $29(50.0)$ & $24(40.7)$ & \\
\hline 2 & $7(12.1)$ & 13(21.7) & & $14(24.1)$ & 20(33.9) & \\
\hline 3 & $3(5.2)$ & $8(13.3)$ & & $1(1.7)$ & $4(6.8)$ & \\
\hline 4 & $0(0)$ & $1(1.7)$ & & $0(0)$ & $0(0)$ & \\
\hline \multicolumn{2}{|l|}{ Neutropenia } & & 0.018 & & & 0.033 \\
\hline 0 & $19(32.8)$ & 17(28.3) & & $13(22.4)$ & $9(15.3)$ & \\
\hline 1 & $27(46.6)$ & 19(31.7) & & $27(46.6)$ & $20(37.3)$ & \\
\hline 2 & $9(15.5)$ & 11(18.3) & & $14(24.1)$ & $22(37.3)$ & \\
\hline 3 & $3(5.2)$ & $9(15.0)$ & & $4(6.9)$ & $6(10.2)$ & \\
\hline 4 & $0(0)$ & $4(6.7)$ & & $0(0)$ & $2(3.4)$ & \\
\hline \multicolumn{2}{|l|}{ Anemia } & & 0.247 & & & 0.049 \\
\hline 0 & $49(84.5)$ & $47(78.3)$ & & $42(72.4)$ & $33(56.9)$ & \\
\hline 1 & $9(15.5)$ & $11(18.3)$ & & 16(27.6) & 23(39.7) & \\
\hline 2 & $0(0)$ & $2(3.3)$ & & $0(0)$ & $2(3.4)$ & \\
\hline \multicolumn{2}{|l|}{ Thrombocytopenia } & & 0.452 & & & 0.532 \\
\hline 0 & 53(91.4) & 53(88.3) & & $55(94.8)$ & $55(93.2)$ & \\
\hline 1 & $5(8.6)$ & $6(10.0)$ & & $3(5.2)$ & $3(5.1)$ & \\
\hline 2 & $0(0)$ & $1(0.8)$ & & $0(0)$ & $1(1.7)$ & \\
\hline \multicolumn{2}{|c|}{ Liver function damage } & & 0.275 & & & 0.178 \\
\hline 0 & $49(84.5)$ & $43(71.7)$ & & $46(79.3)$ & $41(69.5)$ & \\
\hline 1 & $7(12.1)$ & 15(21.6) & & 12(20.7) & $17(28.8)$ & \\
\hline 2 & $1(1.7)$ & $2(3.3)$ & & $0(0)$ & $1(1.7)$ & \\
\hline 3 & $1(1.7)$ & $0(0)$ & & $0(0)$ & $0(0)$ & \\
\hline \multicolumn{2}{|c|}{ Renal function damage } & & 0.166 & & & 0.254 \\
\hline 0 & $55(94.8)$ & 53(88.3) & & 55(94.8) & $53(88.3)$ & \\
\hline 1 & $3(5.2)$ & $6(10.0)$ & & $3(5.2)$ & $6(10.0)$ & \\
\hline 2 & $0(0)$ & $1(1.7)$ & & $0(0)$ & $0(0)$ & \\
\hline \multicolumn{2}{|c|}{ Gastrointestinal reaction } & & 0.032 & & & 0.037 \\
\hline 0 & $8(13.8)$ & $3(5.0)$ & & $9(15.5)$ & $4(6.8)$ & \\
\hline 1 & $38(65.5)$ & $37(61.7)$ & & $32(55.2)$ & $28(47.5)$ & \\
\hline 2 & $11(19.0)$ & $16(26.7)$ & & $16(27.6)$ & $25(42.4)$ & \\
\hline 3 & $1(1.7)$ & $4(6.7)$ & & $1(1.7)$ & $2(3.4)$ & \\
\hline \multicolumn{2}{|l|}{ Oral mucositis } & & 0.099 & & & 0.020 \\
\hline 0 & $55(94.8)$ & $51(85.0)$ & & $12(20.7)$ & $6(10.2)$ & \\
\hline 1 & $2(3.4)$ & $6(10.0)$ & & $27(46.6)$ & 23(39.0) & \\
\hline 2 & $1(1.7)$ & $3(5.0)$ & & 19(32.8) & $28(47.5)$ & \\
\hline 3 & $0(0)$ & $0(0)$ & & $0(0)$ & $2(3.4)$ & \\
\hline \multicolumn{2}{|l|}{ skin rash } & & 0.012 & & & \\
\hline 0 & $52(89.7)$ & $60(100)$ & & - & - & - \\
\hline 1 & $6(10.3)$ & $0(0)$ & & - & - & - \\
\hline
\end{tabular}


Table 3 Adverse reactions in the two groups (Continued)

\begin{tabular}{|c|c|c|c|c|c|c|}
\hline \multirow[t]{2}{*}{ Adverse reaction } & \multicolumn{3}{|l|}{$\begin{array}{l}\text { Induction therapy stage } \\
(n=118)(\%)\end{array}$} & \multicolumn{3}{|l|}{$\begin{array}{l}\text { Concurrent radiochemotherapy stage } \\
(n=117)(\%)\end{array}$} \\
\hline & NPF group $(n=58)$ & DPF group $(n=60)$ & $P$ & NPF group $(n=58)$ & DPF group $(n=59)$ & $P$ \\
\hline \multicolumn{6}{|c|}{ Radiation skin reaction } & 0.035 \\
\hline 0 & - & - & & $8(13.8)$ & $6(10.2)$ & \\
\hline 1 & - & - & & $34(58.6)$ & $25(42.4)$ & \\
\hline 2 & - & & & 16(27.6) & $26(44.1)$ & \\
\hline 3 & - & - & & $0(0)$ & $2(3.4)$ & \\
\hline
\end{tabular}

hamper the application of induction chemotherapy and follow-up concurrent radiochemotherapy to a certain extent. It was reported that the incidence of grade 3-4 neutropenia in taxane-based induction therapy is significantly higher than that of non-taxane containing regimens $(39.1 \%$ vs $16.1 \%, \mathrm{OR}=3.62,95 \% \mathrm{CI} 2.42-5.40, P<$ $0.001, z=6.29$ ) [26]. The incidence rates of neutropenia and stomatitis were 42 and $41 \%$, respectively, after addition of the DPF regimen based on CCRT, which affected compliance with IC and subsequent CCRT. Adding anti-EGFR therapy to standard therapy (radiotherapy or radiochemotherapy) significantly increases $\mathrm{OS}(\mathrm{HR}=$ $0.51 ; 95 \% \mathrm{CI}, 0.39-0.66)$ and DFS $(\mathrm{HR}=0.68 ; 95 \% \mathrm{CI}$, 0.54-0.86); meanwhile, systemic toxicity significantly decreases when EGFR therapy replaces cytotoxic drugs at the time of radiotherapy [27]. In NPC patients administered IMRT, CTX/NTZ combined with induction therapy can reduce severe toxicity and achieve better survival benefit compared with CTX/NTZ applied in concurrent radiotherapy [28]. These findings suggest that anti-EGFR monoclonal antibody combined with an appropriate intensity of chemotherapy may constitute an effective induction therapy to ensure or increase efficacy while markedly alleviating toxic reactions. Because of humanization, NTZ has better tolerance in nasopharyngeal carcinoma treatment $[21,29]$. Compared with CTX, NTZ significantly improves skin rash $(P<0.001)$, oral mucositis $(P<0.001)$ and weight loss $(P<0.008)[29,30]$. In this study, addition of humanized anti-EGFR monoclonal antibody created conditions to reduce the intensity of induction chemotherapy; therefore, systemic toxicity and gastrointestinal reactions during induction

Table 4 Expression of EGFR in both patient groups

\begin{tabular}{llll}
\hline EGFR expression & NPF group (\%) & DPF group (\%) & Total (\%) \\
\hline $0-4 \%$ & $3(5.2)$ & $3(5.0)$ & $6(5.1)$ \\
$5-24 \%$ & $10(17.2)$ & $3(5.0)$ & $13(17.2)$ \\
$25-49 \%$ & $9(15.5)$ & $8(13.3)$ & $17(14.4)$ \\
$50-74 \%$ & $26(44.8)$ & $29(48.3)$ & $55(46.6)$ \\
$75-100 \%$ & $10(17.2)$ & $17(28.3)$ & $27(22.9)$ \\
$P$ & 0.058 & & \\
\hline
\end{tabular}

chemotherapy were effectively controlled. Compared with DPF, the NPF regimen effectively alleviated neutropenia $(P=0.018)$ and gastrointestinal reactions $(P=$ 0.032 ) in the induction phase, while use of EGFR monoclonal antibody in human chemotherapy did not increase mucosal-related toxicity. Toxicity control during induction therapy could also effectively improve patient tolerance during follow-up concurrent radiochemotherapy. Therefore, neutropenia, anemia, gastrointestinal reaction, oral mucositis and radiation dermatitis in the NPF group were significantly improved compared with the DPF group $(P=0.033, P=0.049, P=0.037, P=0.020$ and $P=0.035$, respectively), which greatly improved the tolerance of NPF patients to whole course treatment and solved key problems that restrict the clinical application of induction chemotherapy which could create conditions for improved prognosis.

The main limitation of this study is that it only performed short-term evaluation of efficacy and adverse reactions. Therefore, long-term evaluation of the NPF regimen deserves further investigation, and would further assess the clinical significance of this regimen. In addition, the latest research shows that gemcitabine and cisplatin as induction chemotherapy added to chemoradiotherapy significantly improved LRFS and OS among patients with locoregionally advanced nasopharyngeal carcinoma [31]. Therefore, a comparison of larger sample sizes with new induced chemotherapy regimens may better confirm the role of NPF schemes.

Table 5 Correlation between EGFR expression and the curative effect of induction therapy

\begin{tabular}{|c|c|c|c|c|c|c|c|c|c|}
\hline \multirow{2}{*}{$\begin{array}{l}\text { EGFR } \\
\text { expression }\end{array}$} & \multicolumn{4}{|c|}{ NPF group $(n=58)$} & \multicolumn{4}{|c|}{ DPF group $(n=60)$} & \multirow[b]{2}{*}{$P$} \\
\hline & $C R$ & PR & SD & PD & $C R$ & PR & SD & PD & \\
\hline $0-4 \%$ & 0 & 1 & 1 & 0 & 0 & 3 & 0 & 0 & \\
\hline $5-24 \%$ & 0 & 4 & 6 & 0 & 0 & 1 & 2 & 0 & \\
\hline $25-49 \%$ & 1 & 7 & 1 & 0 & 0 & 4 & 4 & 0 & 0.033 \\
\hline $50-74 \%$ & 0 & 18 & 8 & 0 & 0 & 23 & 6 & 1 & \\
\hline $75-100 \%$ & 1 & 9 & 1 & 0 & 0 & 6 & 10 & 0 & \\
\hline$P$ & \multicolumn{4}{|c|}{0.015} & \multicolumn{4}{|c|}{0.09} & 1 \\
\hline
\end{tabular}




\section{Conclusion}

In conclusion, compared with DPF induction chemotherapy for locally advanced NPC cases receiving followup cisplatin and concurrent IMRT, nimotuzumab combined with PF as induction chemotherapy has better lymph node response rate, less adverse reactions, and better induction therapy and concurrent radiochemotherapy tolerance compared with the DPF regimen. Therefore, the NPF regimen may become a new choice for induction therapy of locally advanced nasopharyngeal cancer.

\section{Abbreviations \\ CCRT: Concurrent radiotherapy and chemotherapy; CR: Complete response; CTX: Cetuximab; DC: Distance control; DFS: Disease-free survival; DMFS: Distant metastasis-free survival; DPF: Docetaxel/PF; EGFR: Epidermal growth factor receptor; IC: Induction chemotherapy; IMRT: Intensity modulated radiation therapy; LRFS: Locoregional recurrence free survival; MR: Magnetic resonance; NPC: Nasopharyngeal carcinoma; NPF: Nimotuzumab/PF; NTZ: Nimotuzumab; OS: Overall survival, PD: Progressive disease; PF: Plus 5-fluorouracil; PFS: Progression-free survival; PR: Partial response; PTV: Planning target volume; RECIST: Response Evaluation Criteria in Solid Tumors; RR: Response rate; SD: Stable disease}

\section{Acknowledgements}

We would like to thank all the patients and the personnel involved in this study. We are also grateful to Professors Min Yi and Wenhua Li at the Pathology Department, the Fourth Affiliated Hospital of Guangxi Medical University, for help in pathologic assessment. This study adheres to CONSORT guidelines.

\section{Authors' contributions}

YL conceived and coordinated the study, designed, performed and analyzed the experiments, wrote the paper. DC, JL, JG, ZL, RW, WL, CH, XN, ML and $\mathrm{HH}$ carried out the data collection, data analysis, and revised the paper. All authors reviewed the results and approved the final version of the manuscript.

\section{Funding}

This work was supported by Liuzhou City Science and technology research projects (2015 J030512), Liuzhou City Science and technology research projects (2016G020203) and Department of Health of Guangxi Zhuang Autonomous Region Self-raised Funds Project (Z2016167), Department of Health of Guangxi Zhuang Autonomous Region Self-raised Funds Project (Z20170895) in the study design, collection and analysis of data.

\section{Availability of data and materials}

The data set supporting the results of this article are included within the article.

\section{Ethics approval and consent to participate}

All procedures performed in studies involving human participants were in accordance with the ethical standards of the institutional and/or national research committee and with the Ethics Committee of the Fourth Affiliated Hospital of Guangxi Medical University (PJK2015201). Written informed consent was obtained from all individual participants included in the study.

\section{Consent for publication}

\section{Not applicable.}

\section{Competing interests}

The authors declare that they have no competing interests.

\section{Author details}

${ }^{1}$ Department of Oncology, the Fourth Affiliated Hospital of Guangxi Medical University, Liuzhou, China. ${ }^{2}$ Department of Radiotherapy, Wuzhou Red Cross Hospital, Wuzhou, China. ${ }^{3}$ Department of Radiotherapy, Liuzhou People's Hospital, Liuzhou, China. ${ }^{4}$ Department of Radiotherapy, the First Affiliated
Hospital of Guangxi Medical University, Nanning, China. ${ }^{5}$ Department of Radiotherapy, the Second Affiliated Hospital of Guangxi Medical University, Nanning, China. ${ }^{6}$ Department of Oncology, the Second People's Hospital of Nanning, Nanning, China. ${ }^{7}$ Department of Oncology, Liuzhou Traditional Chinese Medical Hospital, Liuzhou, China. ${ }^{8}$ Department of Radiotherapy, the Affiliated Hospital of Guilin Medical College, Guilin, China.

Received: 23 April 2019 Accepted: 12 December 2019

Published online: 30 December 2019

\section{References}

1. Liang SB, Wang Y, Hu XF, He SS, Yang XL, Liu LZ, et al. Survival and toxicities of IMRT based on the RTOG protocols in patients with nasopharyngeal carcinoma from the endemic regions of China. J Cancer. 2017;8:3718-24.

2. Bhattacharyya T, Babu G, Kainickal CT. Current role of chemotherapy in nonmetastatic nasopharyngeal Cancer. J Oncol. 2018;2018:3725837.

3. Yan $\mathrm{H}, \mathrm{CaO} X$, Wang J. Application of intensity-modulated radiation therapy in the treatment of nasopharyngeal carcinoma. Oncol Lett. 2017;14:7773-6.

4. Peng $H$, Chen L, Zhang J, Li WF, Mao YP, Zhang Y, et al. Induction chemotherapy improved Long-term outcomes of patients with Locoregionally advanced nasopharyngeal carcinoma: a propensity matched analysis of 5-year survival outcomes in the era of intensity-modulated radiotherapy. J Cancer. 2017:8:371-7.

5. Ribassin-Majed L, Marguet S, Lee AWM, Ng WT, Ma J, Chan ATC, et al. What is the best treatment of locally advanced nasopharyngeal carcinoma? An individual patient data network meta-analysis. J Clin Oncol. 2017;35:498-505.

6. Chen YP, Tang LL, Yang Q, Poh SS, Hui EP, Chan ATC, et al. Induction chemotherapy plus concurrent Chemoradiotherapy in endemic nasopharyngeal carcinoma: individual patient data pooled analysis of four randomized trials. Clin Cancer Res. 2018;24:1824-33.

7. Lang J, Gao L, Guo Y, Zhao C, Zhang C, Society of H, et al. Comprehensive treatment of squamous cell cancer of head and neck: Chinese expert consensus 2013. Future Oncol. 2014;10:1635-48.

8. Skulsky SL, O'Sullivan B, McArdle O, Leader M, Roche M, Conlon PJ, et al. Review of high-risk features of cutaneous squamous cell carcinoma and discrepancies between the American joint committee on Cancer and NCCN clinical practice guidelines in oncology. Head Neck. 2017;39:578-94.

9. Kim R, Hahn S, Shin J, Ock CY, Kim M, Keam B, et al. The effect of induction chemotherapy using Docetaxel, Cisplatin, and fluorouracil on survival in locally advanced head and neck squamous cell carcinoma: a meta-analysis. Cancer Res Treat. 2016:48:907-16.

10. Zeng Z, Yan RN, Tu L, Wang YY, Chen PR, Luo F, et al. Assessment of concurrent Chemoradiotherapy plus induction chemotherapy in advanced nasopharyngeal carcinoma: Cisplatin, fluorouracil, and Docetaxel versus gemcitabine and Cisplatin. Sci Rep. 2018;8:15581.

11. Sun Y, Li WF, Chen NY, Zhang N, Hu GQ, Xie FY, et al. Induction chemotherapy plus concurrent chemoradiotherapy versus concurrent chemoradiotherapy alone in locoregionally advanced nasopharyngeal carcinoma: a phase 3, multicentre, randomised controlled trial. Lancet Oncol. 2016;17:1509-20.

12. Kawahira M, Yokota $T$, Hamauchi S, Onozawa $Y$, Ogawa H, Onoe T, et al. Survival benefit of adding docetaxel, cisplatin, and 5-fluorouracil induction chemotherapy to concurrent chemoradiotherapy for locally advanced nasopharyngeal carcinoma with nodal stage N2-3. Jpn J Clin Oncol. 2017:47:705-12.

13. Kong L, Zhang Y, Hu C, Guo Y, Lu JJ. Effects of induction docetaxel, platinum, and fluorouracil chemotherapy in patients with stage III or IVA/B nasopharyngeal cancer treated with concurrent chemoradiation therapy: final results of 2 parallel phase 2 clinical trials. Cancer. 2017;123:2258-67.

14. Herbst RS, Shin DM. Monoclonal antibodies to target epidermal growth factor receptor-positive tumors: a new paradigm for cancer therapy. Cancer. 2002;94:1593-611.

15. Sun W, Long G, Wang J, Mei Q, Liu D, Hu G. Prognostic role of epidermal growth factor receptor in nasopharyngeal carcinoma: a meta-analysis. Head Neck. 2014;36:1508-16.

16. Ma X, Huang J, Wu X, Li X, Zhang J, Xue L, et al. Epidermal growth factor receptor could play a prognostic role to predict the outcome of nasopharyngeal carcinoma: a meta-analysis. Cancer Biomark. 2014;14: 267-77. 
17. Ma BB, Poon TC, To KF, Zee B, Mo FK, Chan CM, et al. Prognostic significance of tumor angiogenesis, Ki 67, p53 oncoprotein, epidermal growth factor receptor and HER2 receptor protein expression in undifferentiated nasopharyngeal carcinoma--a prospective study. Head Neck. 2003;25:864-72.

18. Peng H, Tang LL, Liu X, Chen L, Li WF, Mao YP, et al. Anti-epidermal growth factor receptor therapy concurrently with induction chemotherapy in locoregionally advanced nasopharyngeal carcinoma. Cancer Sci. 2018;109: 1609-16.

19. Wang Y, Pan L, Sheng XF, Chen S, Dai JZ. Nimotuzumab, a humanized monoclonal antibody specific for the EGFR, in combination with temozolomide and radiation therapy for newly diagnosed glioblastoma multiforme: first results in Chinese patients. Asia Pac J Clin Oncol. 2016;12: e23-9.

20. Chong DQ, Toh XY, Ho IA, Sia KC, Newman JP, Yulyana Y, et al. Combined treatment of Nimotuzumab and rapamycin is effective against temozolomide-resistant human gliomas regardless of the EGFR mutation status. BMC Cancer. 2015;15:255.

21. Wang F, Jiang C, Ye Z, Sun Q, Liu T, Xu M, et al. Efficacy and safety of nimotuzumab with neoadjuvant chemotherapy followed by concurrent chemoradiotherapy for locoregionally advanced nasopharyngeal carcinoma. Oncotarget. 2017;8:75544-56.

22. Liu ZG, Zhao Y, Tang J, Zhou YJ, Yang WJ, Qiu YF, et al. Nimotuzumab combined with concurrent chemoradiotherapy in locally advanced nasopharyngeal carcinoma: a retrospective analysis. Oncotarget. 2016;7: 24429-35.

23. Huang J, Zou Q, Qian D, Zhou L, Yang B, Chu J, et al. Intensity-modulated radiotherapy plus nimotuzumab with or without concurrent chemotherapy for patients with locally advanced nasopharyngeal carcinoma. Onco Targets Ther. 2017;10:5835-41.

24. Chua DT, Nicholls JM, Sham JS, Au GK. Prognostic value of epidermal growth factor receptor expression in patients with advanced stage nasopharyngeal carcinoma treated with induction chemotherapy and radiotherapy. Int J Radiat Oncol Biol Phys. 2004;59:11-20.

25. Ou D, Blanchard P, El KC, De FF, Even C, Levy A, et al. Induction chemotherapy with docetaxel, cisplatin and fluorouracil followed by concurrent chemoradiotherapy or chemoradiotherapy alone in locally advanced non-endemic nasopharyngeal carcinoma. Oral Oncol. 2016;62: $114-21$.

26. Tian $R$, Ye HX, Zhang BG, Gu DY, Zhang BW, Teng ZP, et al. Use of taxanecontaining induction chemotherapy in combination with concurrent chemoradiotherapy in Chinese patients with locally advanced nasopharyngeal carcinoma: a meta-analysis. Onco Targets Ther. 2015;8: 3255-63.

27. Peng $L$, Liu $Z L, X u C$, Tang $L L$, Liu $X$, Lin $A H$, et al. The efficacy and safety of anti-epidermal growth factor receptor monoclonal antibodies in nasopharyngeal carcinoma: literature-based meta-analyses. J Cancer. 2018;9: 4510-20.

28. Peng H, Tang LL, Liu X, Chen L, Li WF, Mao YP, et al. Anti-EGFR targeted therapy delivered before versus during radiotherapy in locoregionally advanced nasopharyngeal carcinoma: a big-data, intelligence platformbased analysis. BMC Cancer. 2018;18:323.

29. Lin M, You R, Liu YP, Zhang YN, Zhang HJ, Zou X, et al. Beneficial effects of anti-EGFR agents, Cetuximab or Nimotuzumab, in combination with concurrent chemoradiotherapy in advanced nasopharyngeal carcinoma. Oral Oncol. 2018;80:1-8.

30. You R, Hua YJ, Liu YP, Yang Q, Zhang YN, Li JB, et al. Concurrent Chemoradiotherapy with or without anti-EGFR-targeted treatment for stage II-IVb nasopharyngeal carcinoma: retrospective analysis with a large cohort and Long follow-up. Theranostics. 2017;7:2314-24.

31. Zhang Y, Chen L, Hu GQ, Zhang N, Zhu XD, Yang KY, et al. Gemcitabine and Cisplatin induction chemotherapy in nasopharyngeal carcinoma. N Engl J Med. 2019;381:1124-35.

\section{Publisher's Note}

Springer Nature remains neutral with regard to jurisdictional claims in published maps and institutional affiliations.

Ready to submit your research? Choose BMC and benefit from:

- fast, convenient online submission

- thorough peer review by experienced researchers in your field

- rapid publication on acceptance

- support for research data, including large and complex data types

- gold Open Access which fosters wider collaboration and increased citations

- maximum visibility for your research: over $100 \mathrm{M}$ website views per year

At BMC, research is always in progress.

Learn more biomedcentral.com/submissions 